\title{
Masseter Treatment with Botulinum Toxin A for parafunctions and quadralized patients
}

Authors:

Redaelli A. ${ }^{1}$

Battistella M. $^{2}$

\section{Affiliations: \\ 1 Department of phlebology and aesthetic medicine, Visconti di Mondrone Medical Center, Milan, Italy \\ 2 Clinical Research Coordi- nator; Biobasic Europe, Milan, Italy.}

\section{Corresponding address:}

Battistella M.

E-mail:

melaniabattistella@gmail.com

\section{DISCLOSURE:}

Redaelli Alessio is a representative and trainer for Ipsen and Filorga companies. His books have been published by OEO Firenze.

Battistella Melania is a representative and trainer for General Project and Wavemed companies.

\section{KEYWORDS:}

Botulinum toxin A, masseter, bruxism, masseter reduction, quadralized patients, masseter hypertrophy

\section{Abstract \\ INTRODUCTION}

Botulinum toxin type A (BTxA) has gained worldwide popularity due to its ability to improve the lower facial contours and treat parafunctions.

\section{MATERIALS AND METHODS}

Various analyses have been conducted through Medline searches for "Botulinum", "masseter" and "bruxism" in order to investigate the different techniques used to inject the masseter muscle with Botulinum toxin. The authors' experiences and techniques are fully explained regarding both functional pathologies and masseter size reduction. Personal guidelines to help physicians achieve the best outcomes for each application are outlined.

Even if a definitive consensus on the use of Botulinum toxin for masseter muscle treatment cannot be reached, the authors recommend, due to their experience, three injection sites per side of the face for masseter hypertrophy and four injection sites per side for the redefinition of the inferior third of the face. Fewer units are needed to inhibit bruxism, while more units are needed in order to achieve a reduction in muscle size.

\section{RESULTS}

Results have been very good and patient satisfaction is usually very high. No side effects have been experienced as of the writing of this report.

\section{DISCUSSION}

BTXA can be used as a therapeutic agent in bruxism patients and has been widely accepted as a cosmetic agent in recent years, helping to re-establish a balance in the contours of quadralized patients due to its positive clinical outcomes. Other clinical investigations are needed to improve the long-term effectiveness of Botulinum toxin.

\section{CONCLUSION}

Botulinum toxin can be considered a safe and effective technique to treat related masseter parafunctions and to improve the shape of the lower face in quadralized patients. 


\section{INTRODUCTION}

Bruxism is a serious psychophysiological disorder and a common clinical issue in dentistry: it is defined as the clenching, grinding, gnashing and tapping of the teeth. It is often classified as either diurnal (occurring while the individual is awake) or nocturnal (occurring while the individual is sleeping).

It is also well known that bruxism causes tooth displacement, TMJ disorders and alterations in periodontal sensitivity.

A variety of therapeutic options have been proposed for bruxism (functional and drug therapy) even though there is no unanimous consensus on the best treatment. Botulinum Toxin type A (BTxA) is a powerful known biological toxin - safe and effective - which has long been used for different purposes in medicine. In modern dentistry, BTxA technique is also used to control masticatory muscles and postsurgical pain, in particular the temporalis and the masseter, i.e., before and after dental implants ${ }^{(1,2)}$. The authors' experiences and casistic report on bruxism were published some years ago, detailing excellent results and zero side effects ${ }^{(3)}$.

In cosmetic medicine, its use is indicated to reduce the volume of the masseter muscle ${ }^{(4)}$ and to improve the aesthetics of the face and the expressive wrinkles $^{(5,6)}$. In fact, aesthetic canons of beauty continue to influence many cultures, so triangular and heart shaped faces are still considered delicate and fascinating compared to quadralized faces with enlarged lower contours.

This is why the authors normally follow the rule that inter-jugal distance should be $82 \%$ of bizygomatic distance. Assuming that the bizygomatic distance is $100 \%$, quadralized patients have a very similar bigonian distance. In rare patients, most often males, the bigonian distance is higher than the bizygomatic distance.
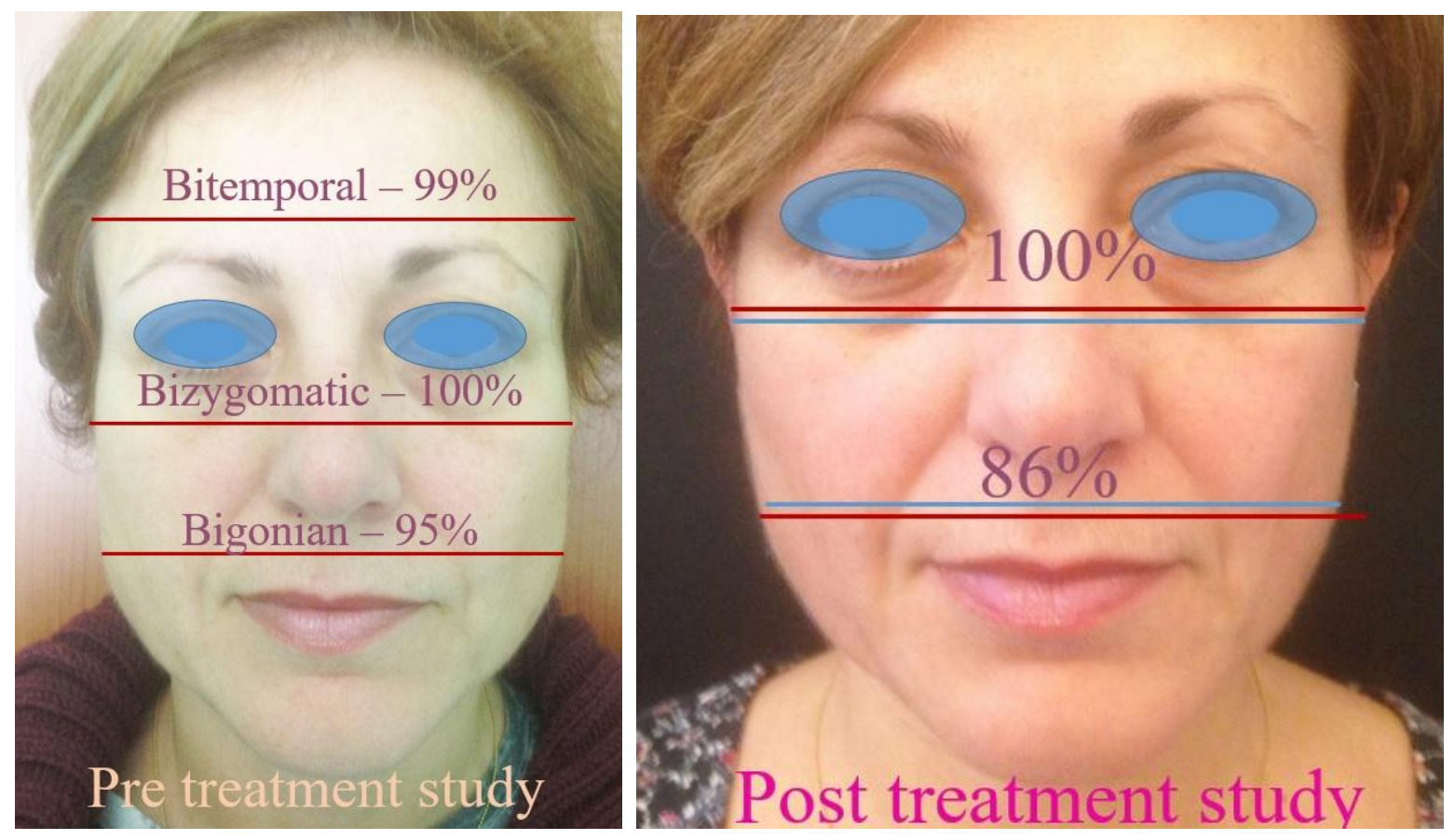

Figure 1: in (a) pre-treatment and in (b) 30 days post-treatment. Assuming that the bizygomatic distance is $100 \%$, it is easy to assess the improvement of the bigonian distance 
A wide lower third of the face is caused by many factors, but primarily by prominent mandibular angles and muscle hypertrophy. There is no consensus on the units required to obtain positive results in bruxers.

The purpose of this article is to recommend the best dosage to use in both cosmetic and functional masseter treatments, based on the evaluation of the literature and the authors' experience.

\section{MATERIALS AND METHODS}

The authors revised the literature using Medline as their main source, inserting "botulinum toxin," "bruxism", and "masseter" as search criteria. All the studies responding to the criteria have been taken into consideration in an attempt to establish simple guidelines with the aim of suggesting a standard BTxA administration that could achieve better aesthetic results for the lower facial contour and for parafunction control.

The authors have used Abobotulinum Toxin A in their day-to-day practice for the last 10 years. In Italy, the commercial name of Abobotulinum Toxin A (AboTA) is Azzalure and for Bruxers the average dilution used is $0,75 \mathrm{ml}$ of normal saline (slightly higher than the dose used for aesthetic purposes). In this dilution ( $3 \mathrm{ml}$ for $500 \mathrm{IU}$ Dysport), $5 \mathrm{U}$ are in $0,03 \mathrm{ml}$ and $20 \mathrm{U}$ in $0,12 \mathrm{ml}$. For Bruxers, 3 sites are injected with a total of $35 \mathrm{UI}=0,21 \mathrm{ml}(10+10$ in the lower sites and $15 \mathrm{U}$ in the highest) (Fig.1) ${ }^{(3)}$ while in quadralized patients, where the reduction of muscle size is the goal, we normally inject 4 sites $(20 \mathrm{U}$ x point $=0,12$ $\mathrm{ml})$ for a total of $80 \mathrm{U}(0,48 \mathrm{ml})$ per side (Fig.2).
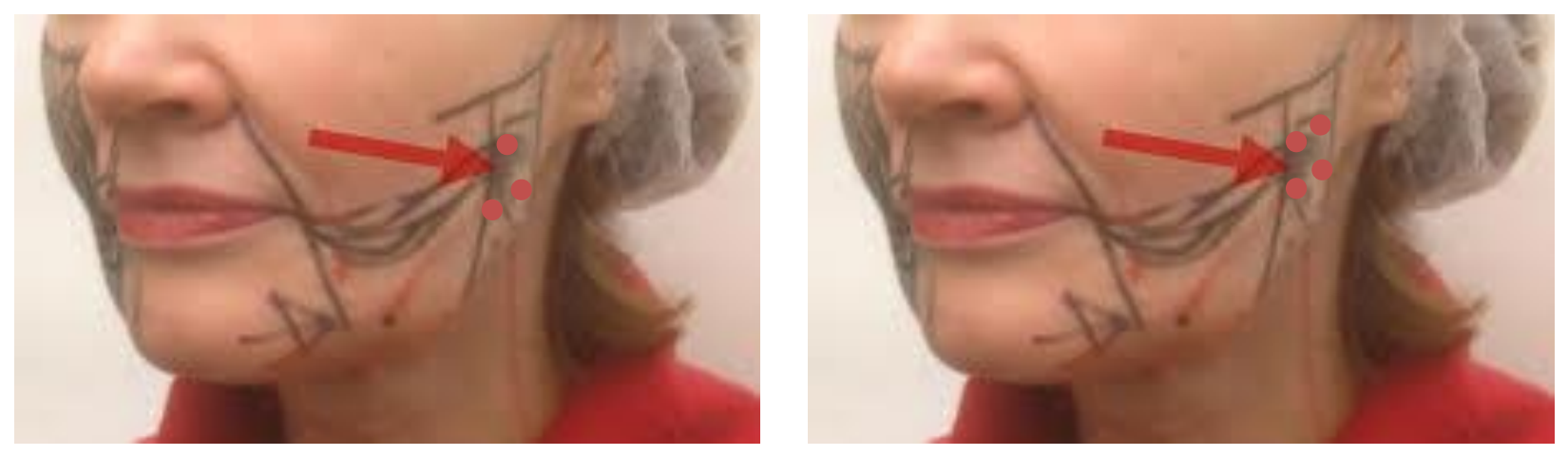

Figure 2: in (a) the injection sites for bruxism and in (b) those for masseter hypertrophy

The ideal botulinum injection site in the masseter is a rectangular area precisely in the center of the muscle: to avoid Stenson's Duct we remain always at least 1 $\mathrm{cm}$ below a line from the Tragus to the upper lip. Then we accurately map the masseter muscle in the anterior and posterior edge by asking the patient to clench their teeth. We select 3 or 4 sites precisely in the center of the muscle according to this reference. These injections must be done in a particular way: the needle is inserted while the patient clenches their teeth and then, as they relax, we inject the dosage as we push the needle into the muscle. In this way, we can avoid the diffusion of the toxin into the Risorius muscle, which is superficial and sometimes presents laterally in parotideal fascia - very close to the masseter.

To perform this technique:

- reconstitute the toxin with the suggested dilution (normally $3 \mathrm{ml}$ normal saline for 500U AboTA),

- aspirate the correct amount of Units in a $0,5 \mathrm{ml}$ insulin syringe with a 


\section{G x 8mm needle}

- ask the patient to simulate biting in order to identify masseter muscle mass

- palpate the muscle to identify the major contraction points

- mark three or four sites, as shown in Figure 2, according to this indication

- insert the $30 \mathrm{G} 8 \mathrm{~mm}$ needle in the center of the contracted muscle

- ask the patient to relax, applying light pressure so that the needle remains in the center of the muscle

- inject the units into each site

\section{RESULTS}

The efficacy of BTxA in reducing the volume of the masseter muscle has been proven by many studies using different injection protocols to confirm its efficacy beyond the results seen in photographs and to attain an assessment of patient satisfaction.

Initially, physicians were primarily interested in analyzing the expression of the proteins involved in the process of muscle reduction in animal (7) and human specimens. ${ }^{(8)}$

Many authors have investigated the effects of BTxA using electromyography (EMG) in animal subjects ${ }^{(9)}$, confirming that masseter muscle activity measured by EMG was immediately decreased after a BTX-A injection ${ }^{(3)}$.

Based on these results, other studies have been conducted using ultrasound (10) and a 3D computed tomography (11) as powerful tools for guiding the injections into the muscles, contributing to a more personalized procedure, better cosmetic results, and helping to avoid potential complications.

Technology, such as the threedimensional laser scanning ${ }^{(12)}$, has been used various times to demonstrate clearly and statistically significant post-injection masseter reduction.

There is still some controversy regarding the injection sites and the quantity of toxin that must be used. Some studies reported five intra-muscle injection sites on both sides using a 29 gauge needle (13); others, conducted on facial dissections suggest three sites per side using different dosages of the toxin. ${ }^{(14,15)}$

Some authors have found that the final measurement of muscle mass following intermittently repeated treatments resulted in a long-lasting reduction. ${ }^{(16)}$

Based on this knowledge, the authors have - over time - refined their techniques for treating masseter hypertrophy during their practice. Regarding lower face contour remodeling with AboTA, they suggest some simple steps as the ideal approach for masseter treatment, as previously outlined.

Masseter hypertrophy can be symmetrical or asymmetrical. As mentioned above, it is possible to treat this aesthetic complaint modulating the quantity of botulinum toxin injection, considering the different needs of each patient.

As always, a strong knowledge of anatomy is essential as is an understanding of the physical structures if the aim is to administer an effective botulinum toxin injection and avoid a potentially iatrogenic unnatural facial expression. Investigations have been performed with the aim of analyzing anatomical landmarks so as to define safe injection techniques.

Based on this, the medial part of the masseter muscle represents the first important site in avoiding diffusion into the last lateral fibers of the Risorius muscle, which can lead to asymmetry in facial expression ${ }^{(17,18)}$. This is why great care must be taken when injecting the red area shown in Figure 3, always remembering to stay solely - and deeply - in the masseter muscle. 
But palpation normally allows doctors to find the precise center of the masseter muscle, especially the superficial one oblique from the angle of the mandible to the zygomatic medial bone.

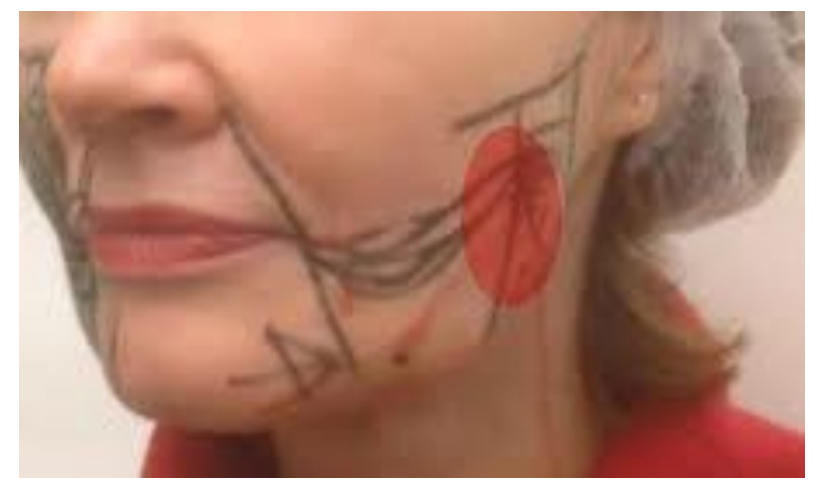

Figure 3: danger zone for toxin diffusion into the risorius muscle

A perfect study and palpation will avoid injections into the parotid gland and into branches of the facial nerves such as the marginal mandibular and buccal. ${ }^{(19)}$

After one treatment, using this dose

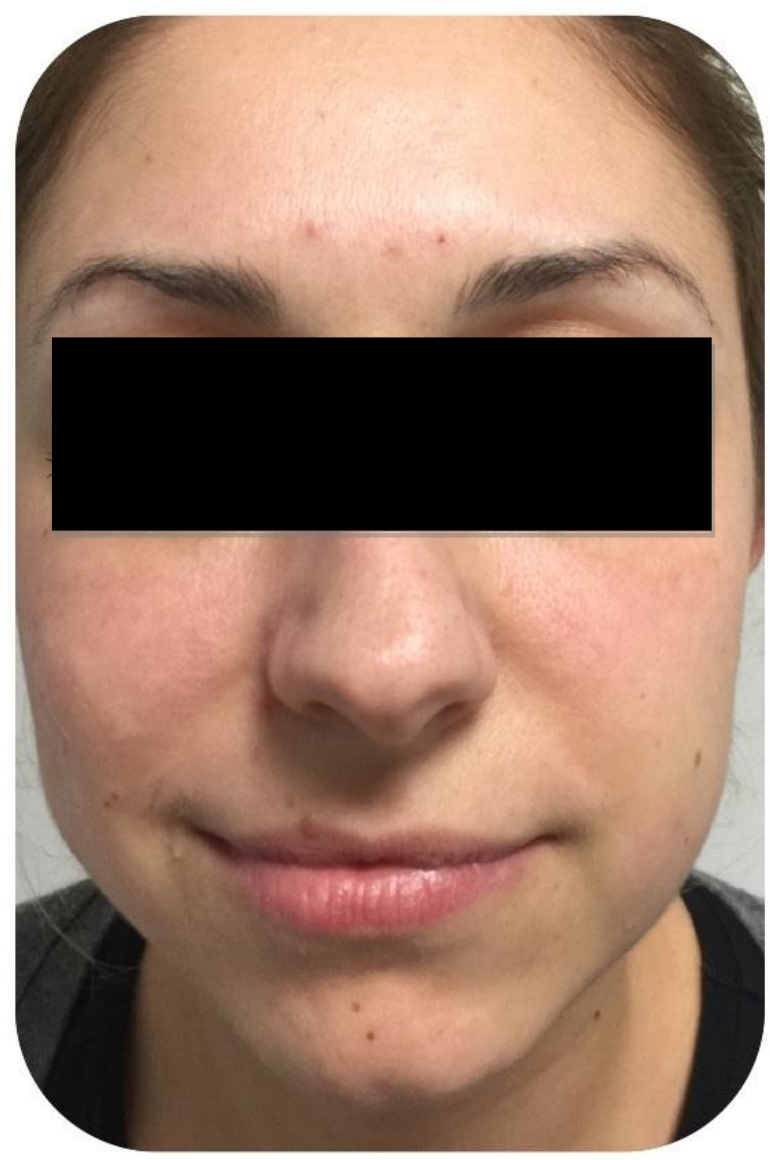

Before and technique, the results consist of a streamlining of the mandibular line that begins appearing more triangular as shown in figures 4 and 5.

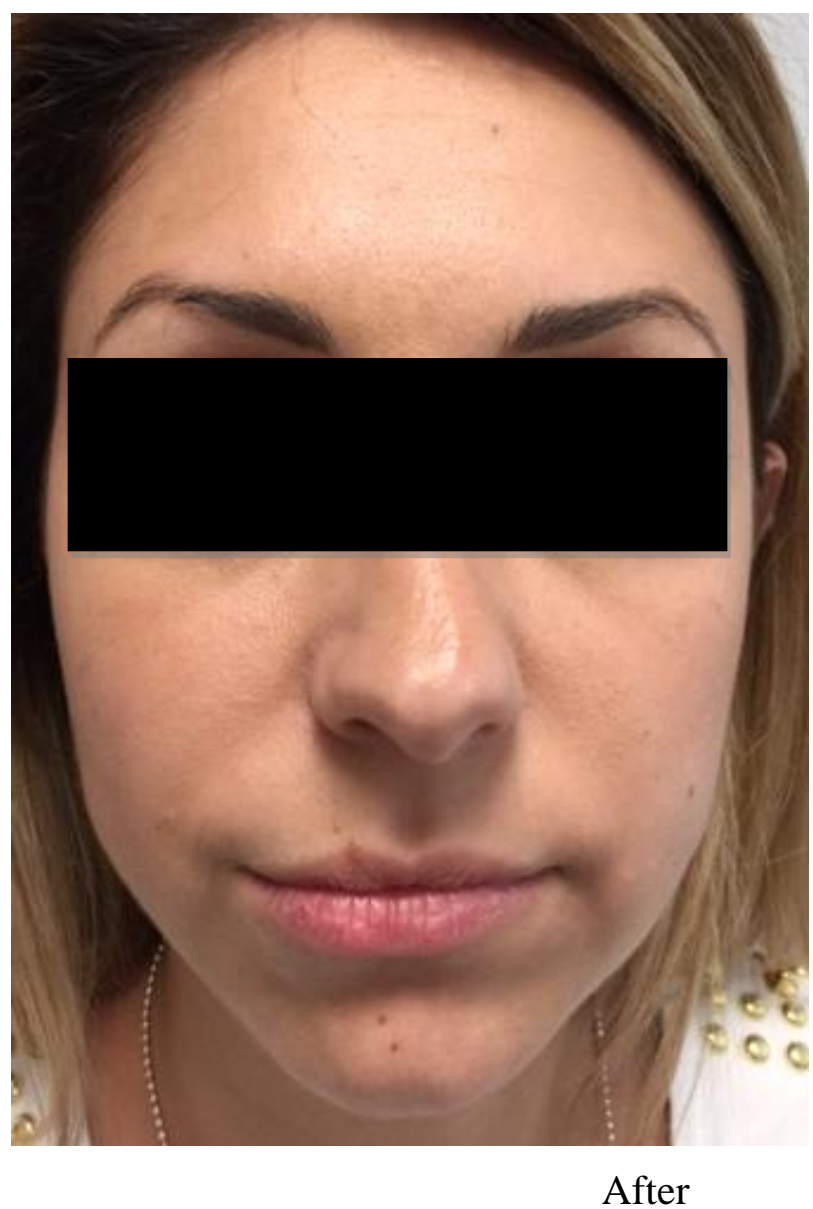

Figure 4 
The post BTxA injection effects usually create a significant change in force balance ${ }^{(20,21)}$, thus the masticatory function declines a bit ${ }^{(22)}$.

Regarding masseter parafunctions, BTxA injection has been also used to treat temporo-mandibular joint dysfunction patients (23) and bruxers, applied to their masseter and temporalis muscles (24) with a high patient satisfaction rate.
In order to maintain the results, the literature also reports some successful strategies like purposefully adjusting the masticatory movements to postpone the masseter muscle re-hypertrophy ${ }^{(25)}$.

No side effects have been experienced in our practice until now, and this is very encouraging as we continue studying the technique to further confirm our results.
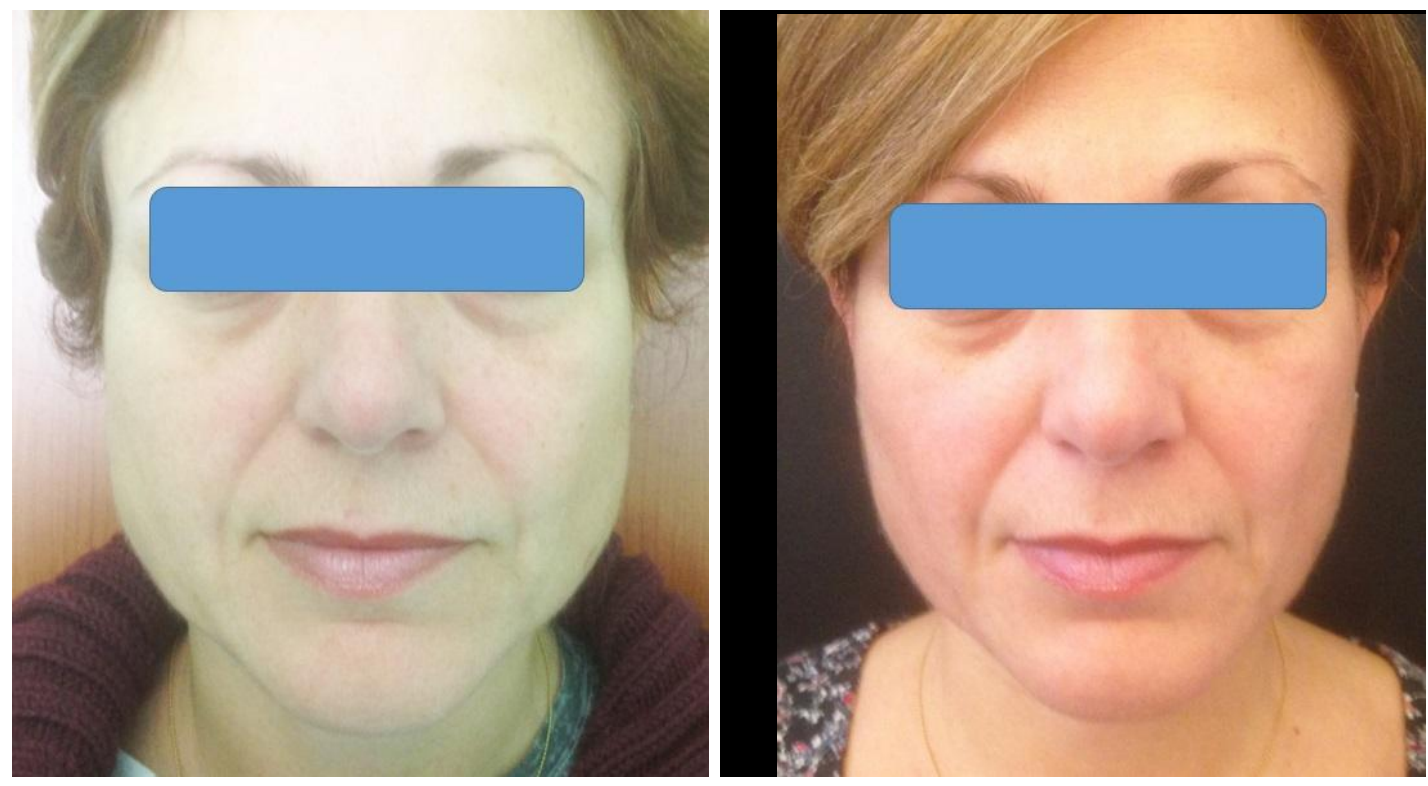

Figure 5: a patient before treatment (a) and after 30 days (b) with just AboTA in masseter muscle

\section{DISCUSSION}

The use of botulinum toxin is a successful minimally invasive procedure and it is used in the management of specific clinical conditions like temporomandibular disorders, bruxism and to achieve volumetric changes in the lower face.

Data obtained by the authors through the use of AboTA for these purposes were first deduced during their daily practice but, however, they are mostly in agreement with the findings of the literature.

The analysis of the lower third of the face after treatment with AboTA was performed by looking at photographs showing the same distance and position. This technique has been proven effective in thinning the masseter muscle in the case of quadralized patients and in bruxers. An improvement in symptoms has always been observed.

Temporary side effects like local edema, ecchymosis, pain at the injection site, headaches, and sometimes dry mouth, were localized, transient and didn't influence the patient's everyday life in relation to the benefits. The standardized amount of Units seems to be effective in the majority of patients but new studies are needed to determine a standardized protocol and consensus and to research an eventual 
strategy for the prolongation of the duration of its effectiveness.

\section{CONCLUSIONS}

Botulinum toxin A can be considered to have a highly convincing profile in terms of both safety and efficacy for the contouring of the lower face and for parafunction treatment. Authors concluded specifically that the use of AboTA at a dosage of $80 \mathrm{U}$ per each masseter is effective in patients who wish for a more triangular lower face shape and at the dose of $35 \mathrm{U}$ per side in those who presented parafunctions. In this last case, results have not been determined by clinical exams or photographs but are only based on patient satisfaction. With increasing clinical experience and expertise, AboTA application can become more precise and personalized in order to guarantee patient satisfaction, with a very low rate of side effects. 


\section{Bibliography:}

(1) Devine M.: Chronic post-surgical pain following the placement of dental implants in the maxilla: A case series. Eur J Oral Implantol. 2016;9 Suppl 1(2):179-86.

(2) Pravnar N.: BOTOX: Broadening the Horizon of Dentistry. J Clin Diagn Res. 2014 Dec; 8(12): ZE25-ZE29

(3) Redaelli A.: Botulinum Toxin A in bruxers. One year experience. Saudi Med J. $2011 \mathrm{Feb} ; 32(2): 156-8$.

(4) Wanitphakdeedecha R: The efficacy of two formulations of botulinum toxin type A for masseter reduction: a split-face comparison study. J Dermatolog Treat. 2016 Dec 6:1-4.

(5) Redaelli A.: cosmetic use of BTxA in the inferior third of the face. Cosmetic Dentistry, 2, 40-47, 2008.

(6) Redaelli A.: Botulinum Toxin A in aesthetic medicine, for Hyperhidrosis and in odontostomatology. $2^{\text {nd }}$ edition, OEO Florence 2015

(7)Park YW1, Kim SG1, Jo YY2S100 and p65 expression are increased in the masseter muscle after botulinum toxin-A injection. Maxillofac Plast Reconstr Surg. 2016 Aug 26;38(1):33.).

(8) Ma F, Zhai Z, Zhu S, Tang S.Ultrastructural changes in human masseter muscles after botulinum neurotoxin a injection. Muscle Nerve. 2017 Feb 10.

(9) Park SY1, Park YW1, Ji YJ2, Park SW3, Kim SG4.Effects of a botulinum toxin type A injection on the masseter muscle: An animal model study. Maxillofac Plast Reconstr Surg. 2015 Feb 24;37(1):10
(10) Quezada-Gaon N1, Wortsman X2, Peñaloza O3, Carrasco JE1.Comparison of clinical marking and ultrasound-guided injection of Botulinum type A toxin into the masseter muscles for treating bruxism and its cosmetic effects. J Cosmet Dermatol. 2016 Sep;15(3):238-44

(11) No YA1, Ahn BH2, Kim BJ1, Kim MN1, Hong CK1.Three-dimensional CT might be a potential evaluation modality in correction of asymmetrical masseter muscle hypertrophy by botulinum toxin injection. $\mathrm{J}$ Cosmet Laser Ther. 2016;18(2):113-5

(12) Lee HH1, Kim ST, Lee KJ, Baik HS.Effect of a second injection of botulinum toxin on lower facial contouring, as evaluated using 3-dimensional laser scanning. Dermatol Surg. 2015 Apr;41(4):439-44

(13) Bhattacharjee K1, Singh M1, Bhattacharjee H1.Extended effect after a single dose of type A botulinum toxin for asymmetric masseter muscle hypertrophy. Indian J Plast Surg. 2015 MayAug;48(2):196-9).

(14) Ahn BK1, Kim YS, Kim HJ, Rho NK, Kim HS.Consensus recommendations on the aesthetic usage of botulinum toxin type A in Asians. Dermatol Surg. 2013 Dec;39(12):1843-60)

(15) K lein, F. Brenner, M. S. Sato,F. Manfron Batista Ros Robert, and Karin Adriane Helme. The administration of a second BoNT-A injection is effective for better aesthetic results for the lower facial contour. An Bras Dermatol. 2014 Nov-Dec; 89(6): 878-884)

(16) Aydil B1, Başaran B, Unsaler S, Süoğlu Y.The use of botulinum toxin type 
A in masseter muscle hypertrophy: longterm effects and lasting improvement. .Kulak Burun Bogaz Ihtis Derg. 2012 SepOct;22(5):249-53

(17) Bae JH1, Choi DY, Lee JG, Seo KK, Tansatit T, Kim HJ.The risorius muscle: anatomic considerations with reference to botulinum neurotoxin injection for masseteric hypertrophy. Dermatol Surg. 2014 Dec;40(12):1334-9)

(18) Klein FH1, Brenner FM2, Sato MS2, Robert FM2, Helmer KA2.Lower facial remodeling with botulinum toxin type A for the treatment of masseter hypertrophy. An Bras Dermatol. 2014 NovDec;89(6):878-84

(19) Kaya B1, Apaydin N2, Loukas M3, Tubbs RS4. The topographic anatomy of the masseteric nerve: A cadaveric study with an emphasis on the effective zone of botulinum toxin A injections in masseter. J Plast Reconstr Aesthet Surg. 2014 Dec;67(12):1663-8

(20) Song JH1, Cho ES1, Kim ST1, Ahn HJ2.Change of distribution and timing of bite force after botulinum toxin type A injection evaluated by a computerized occlusion analysis system. Yonsei Med J. 2014 Jul;55(4):1123-9

(21) Zhang LD1, Liu Q1, Zou DR1, Yu LF2Occlusal force characteristics of masseteric muscles after intramuscular injection of botulinum toxin A(BTX - A)for treatment of temporomandibular disorder. $\mathrm{Br}$ J Oral Maxillofac Surg. 2016 Sep;54(7):73640

(22) Park HU1, Kim BI, Kang SM, Kim ST, Choi JH, Ahn HJChanges in masticatory function after injection of botulinum toxin type A to masticatory muscles. J Oral Rehabil. 2013 Dec;40(12):916-22).

(23) Pihut M1, Ferendiuk E1, Szewczyk M1, Kasprzyk K2, Wieckiewicz M3.The efficiency of botulinum toxin type A for the treatment of masseter muscle pain in patients with temporomandibular joint dysfunction and tension-type headache. J Headache Pain. 2016;17:29

(24) Sevim S1, Kaleağası H2, Fidanc1 H2.Sleep bruxism possibly triggered by multiple sclerosis attacks and treated successfully with botulinum toxin: Report of three cases. Mult Scler Relat Disord. 2015 Sep;4(5):403-5) .

(25) Wei J1, Xu H, Dong J, Li Q, Dai C.Prolonging the duration of masseter muscle reduction by adjusting the masticatory movements after the treatment of masseter muscle hypertrophy with botulinum toxin type a injection. Dermatol Surg. 2015 Jan;41 Suppl 1:S101-9 